\title{
The Modified PID Network of Aeroengine Multivariable Decoupling Control System
}

\author{
J. Ying, H. Rong, J.Y. Dai, F.T. Huang \\ Nondestructive Test Key Laboratory of Ministry Education \\ Nanchang Hangkong University \\ Nanchang 330063, China
}

\begin{abstract}
To solve the decoupling problem in aeroengine multivariable contrl system, in this article,using the modified PID network design the decoupling controller for aeroengine multivariable system. PID network weights using gradient learning algorithm, the network weights fixed more slowly and were easy to fall into local minimum value,so in this paper by means of adding momentum item, improve the efficiency of online learning.The simulation results show that,using this manner design the decoupling controller has high decoupling,low justive time,high precision error and so forth.Using this manner is content to design for the aeroengine controller.
\end{abstract}

Keywords- aeroengine; multivariable; SVM; PIDN; decoupling controller

\section{INTRODUCTION}

Aircraft engine is a kind of strongly nonlinear and complex, time-varying pneumatic thermodynamics system.In the process of their work, will be affected by many uncertain factors, and changes in external conditions, the engine work status and performance will change accordingly, may even lead to the instability of flight. Traditional controller have limitations in the specific control system,when the serious nonlinear controlled object, the mathematical model of uncertainty, the system working point the factors such as dramatic changes,there is difficult to make up for the serious defects of the traditional controller,the effectiveness of its application is limited by a lot of,this causes the people to new control technology and methods are put forward[1, 2].

Proposed in reference [3],traditional PID control method combined with neural network control method of PID neural network controller.PID network is not a simple combination of neural network and PID control law,which will be incorporated in the neural networks, the PID control law so that it has advantages of neural network and PID control.By the simulation experiments show that using PID network to establish the decoupling control of aircraft engine can satisfy the control requirement.

\section{AIRCRAFT ENGINE MODEL}

Assuming that Aircraft engine in a balance of small deviation state variable model is expressed as:

$$
\begin{aligned}
& \dot{x}=A x+B u \\
& y=C x+D u
\end{aligned}
$$

then $\quad x=\left[n_{L}, n_{H}\right]^{T} \quad, \quad u=\left[W_{f}, A_{8}, G_{V F}, G_{V C}\right]^{T}$, $y=\left[n_{L}, n_{H}, P I T, T_{46}\right]^{T}, \quad n_{L}$ and $n_{H}$ is the turbine speed, the main oil is $W_{f}, A_{8}$ as the exhaust area, $G_{V F}$ is fan vane angle, $G_{V C}$ as compressor guide vane angle, $P I T$ is turbine divide than, $T_{46}$ is low pressure turbine inlet temperature.

The working mechanisms of a engine flight envelope is shown in figure 1.Aircraft engine in this area, do not overload, not overtemperature super power.

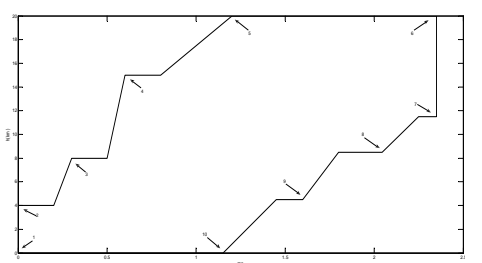

FIGURE I. AIRCRAFT ENGINE FLIGHT ENVELOPE.

Within the flight envelope, any flight state point can use this method to establish small deviation state variable model.Now in flight envelope point 1 (horizon) is used to model as an example.

In the matching point place of $\left(x_{0}, u_{0}, y_{0}\right)$ to $0.02 \mathrm{~s}$ for the cycle,Continue to collect $15 \mathrm{~s}$ system dynamic response data,using the least squares fitting modeling method for fitting.Get the coefficient matrix is as follows:

$$
\begin{aligned}
& A=\left[\begin{array}{cc}
-2.5479 & 3.2784 \\
0.1531 & -3.6167
\end{array}\right] \\
& B=\left[\begin{array}{cccc}
0.89538 & 1.4062 & -1.0934 & 0.4203 \\
0.5797 & 0.2455 & -0.0320 & -0.2827
\end{array}\right] \\
& C=\left[\begin{array}{cc}
1 & 0 \\
0 & 1 \\
-0.9703 & 5.0300 \\
0.3598 & -1.9333
\end{array}\right]
\end{aligned}
$$




$$
D=\left[\begin{array}{cccc}
0 & 0 & 0 & 0 \\
0 & 0 & 0 & 0 \\
-0.1610 & 0.6339 & -0.3326 & 0.5071 \\
0.4930 & 0.0985 & 0.0354 & -0.2729
\end{array}\right]
$$

III. MULTIVARIABLE DECOUPLING CONTROLLER BASED ON MODIFIED PID NETWORK

\section{A. Multivariable Decoupling Controller Based on Modified} PID Network System Structure

From the structure of PID network can be divided into three layer that is input layer, hidden layer and output layer , four input output variable controller is designed in this paper, four variables control network topology structure is as follows:

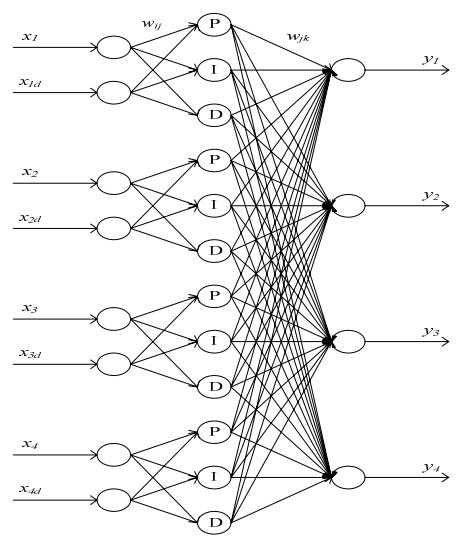

FIGURE II. FOUR VARIABLE PID CONTROL NETWORK TOPOLOGY.

As shown in figure 2, diagram to control the current value of $X_{1}, \ldots, X_{4}, X_{1 d}, \ldots, X_{4 d}$ to control the amount of control target, $y_{1}, \ldots, y_{4}$ for control network control law, $\omega_{i j}$ and $\omega_{j k}$ is the network weights.

Which input layer contains eight neurons, input data $X_{s i}$ is equal to the output data ${ }^{X_{s i}}$, calculating formula is:

$$
x_{s i}(k)=X_{s i}(k)
$$

Hidden layer has twelve neurons, including proportion of four neurons, four integral neurons and differential neurons [5].Its computation formula is:

$$
n e t_{s j}(k)=\sum_{i=1}^{2} \omega_{i j} x_{s i}(k), j=1,2,3
$$

The output of hidden layer neurons computation formula is as follows:

$$
u_{s 1}(k)=n e t_{s 1}(k)
$$

Formula (4) as the proportion of neurons output formula,

$$
u_{s 2}(k)=n e t_{s 2}(k)+u_{s 2}(k-1)
$$

Formula (5) as the integral output neurons,

$$
u_{s 3}(k)=n e t_{s 3}(k)+u_{s 3}(k-1)
$$

Formula (6) for differential output neurons, ${ }^{S}$ is parallel subnet number. $j$ is subnet of hidden layer neurons number; $x_{s i}(k)$ is Each network input layer neurons output; ${ }^{{ }^{i j}}$ Is the network the weights between input layer to hidden layer.

Output layer has four neurons, constitute four-dimensional output, the output of the output layer is the output value of the weighted hidden layer neurons and all, is calculated as follows:

$$
y_{h}(k)=\sum_{s=1}^{4} \sum_{j=1}^{3} \omega_{j k} u_{s j}(k)
$$

Formula (7), $h_{\text {for the output layer neurons number; } s}$ for the sub-network hidden layer neuron number; $u_{s j}(k)$ is the output value of each neuron hidden layer;the connection weights $\omega_{j k}$ hidden layer output layer.

Network PID control in the control process according to the amount of error correction method according to the gradient correction weights, so that the control continues to be close to the amount of control target weights amended as follows:

$$
J=\frac{1}{2} \sum_{k=1}^{4}\left[y_{h}(k)-r(k)\right]^{2}
$$

Of formula (8) is the error calculation formula, $y_{h}$ is the predicted output, $r$ is a control target,

$$
\omega_{j k}(k+1)=\omega_{j k}(k)-\eta \frac{\partial J}{\partial \omega_{j k}}
$$

Formula (9) for the PID network hidden layer to the output layer weights correction formula,

$$
\omega_{i j}(k+1)=\omega_{i j}(k)-\eta \frac{\partial J}{\partial \omega_{i j}}
$$

Equation (10) for the PID input layer to the hidden layer weights correction formula, $\eta$ is learning rate.

PID network weights gradient learning algorithm, network weights corrected slow and easy to fall into local minimum,in this paper, to improve network efficiency by adding momentum learning methods, correction formula PID network weights modified:

$$
\begin{gathered}
\omega_{j k}(k+1)=\omega_{j k}(k)-\eta \frac{\partial J}{\partial \omega_{j k}} \\
+\eta_{1}\left[\omega_{j k}(k)-\omega_{j k}(k-1)\right] \\
\omega_{i j}(k+1)=\omega_{i j}(k)-\eta \frac{\partial J}{\partial \omega_{i j}} \\
+\eta_{1}\left[\omega_{i j}(k)-\omega_{i j}(k-1)\right]
\end{gathered}
$$


Formula (11) and (12) is for after modifying the hidden layer to the output layer weights correction formula and the input layer to the hidden layer weights correction formula,

where $\eta_{1}$ is the learning factor adding momentum.

\section{B. Modified PID Network of Aeroengine Multivariable}

Decoupling Control System Design Principles

In this paper, four variables improved aero-engine small deviation state variable model of the least squares method for the study,to verify the performance of the designed network decoupling PID controller. This is only the choice within the flight envelope height $H=0 \mathrm{~km}, M a=0$ Mach study.

The establishment of four variables aeroengine small deviation state variable model as follows:

$$
\left[\begin{array}{l}
y_{1} \\
y_{2} \\
y_{3} \\
y_{4}
\end{array}\right]=\left[\begin{array}{l}
g_{11}, g_{12}, g_{13}, g_{14} \\
g_{21}, g_{22}, g_{23}, g_{24} \\
g_{31}, g_{32}, g_{33}, g_{34} \\
g_{41}, g_{42}, g_{43}, g_{44}
\end{array}\right]\left[\begin{array}{l}
u_{1} \\
u_{2} \\
u_{3} \\
u_{4}
\end{array}\right]
$$

The mathematical model is described by the following equation $[4,5]$.

$$
\begin{aligned}
& y_{h}(t)=f\left[y_{h}(t-1), \ldots, y_{h}(t-n),\right. \\
& \left(u_{1}(t-1), \ldots, u_{1}\left(t-m_{1}\right)\right), \ldots, \\
& \left.\left(u_{4}(t-1), \ldots, u_{4}\left(t-m_{4}\right)\right)\right]
\end{aligned}
$$

Based on this model PID controller and controlled network system composed of closed loop control system as shown:

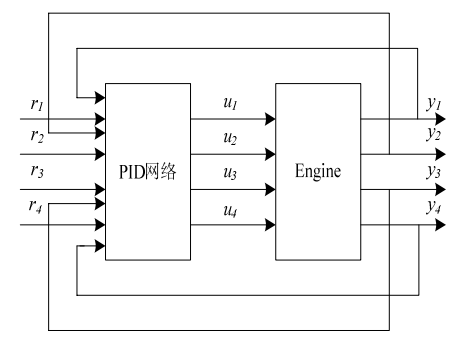

FIGURE III. PID CLOSED LOOP CONTROL SYSTEM NETWORK.

Among them, $r_{1}, \ldots, r_{4}$ is control target control amount, $u_{1}, \ldots, u_{4}$ is controller control law, $y_{1}, \ldots, y_{4}$ is the current value of the control amount predicted value for the system output.

\section{CONTROL SYSTEM PERFORMANCE ANALYSIS}

Shown in figure 5 using the network PID closed loop control system,four variables establish minimum state variable model of aeroengine (SVM) method in accordance with 1.2, the choice within the flight envelope height $H=0 \mathrm{~km}$,Mach $M a=0$ as research subjects to establish a network coupled PID controller to control,certain biaxial turbofan aeroengine model sampling period is $T=20 \mathrm{~ms}$, the number of samples for the $C=200$,network weights $\omega$ initialized to a random number between $0 \sim 0.03$, Control the amount of the initial value is $\left[\begin{array}{llll}0 & 0 & 0 & 0\end{array}\right]$, control objectives for [ $\begin{array}{llll}0.71 & 0.13\end{array}$ 0.690 .31 ],input layer to the hidden layer learning rate is $\eta_{1}=0.006$,hidden layer to the output layer, learning rate is $\eta_{2}=0.001$,the increased momentum learning rate is $\eta_{3}=0.00001$

In this paper, a simulation matlab environment[6], as shown in Figure 5 to establish a network-based PID based on four variables aeroengine aeroengine decoupling control, the simulation as shown in Figure 7

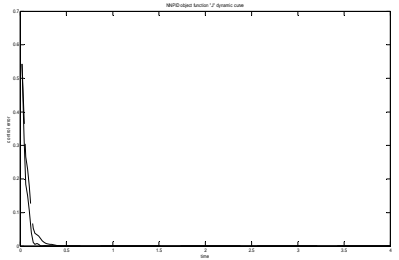

FIGURE IV. CONTROL SYSTEM ERROR CURVE.

The dotted line is no momentum system output error curve shown in Figure 7, the solid line is adding momentum system output error.The simulation results showed that adding network decoupling PID controller momentum, the basic phenomenon of a certain type of biaxial eliminate coupling turbofan aeroengine model, and there is no steady-state error.

\section{CONCLUSIONS}

In this paper, the coupling between a certain type of biaxial turbofan aeroengine control volume and each loop,The improved method of least squares established aeroengine four small deviation variable state variable model for the study,PID network design aeroengine multivariable decoupling controller.Studies have shown that this method is based on the design of the network aeroengine multivariable PID decoupling controller has good decoupling characteristics, can be effectively applied to multivariable decoupling control certain type twin turbofan aircraft engines.

\section{REFERENCES}

[1] Si.Qi.Fan. Aero engine control[M]. Northwestern Polytechnical University, 2008.6

[2] Zhi.Kun.Wu. Study on the design of turbofan engine control system[D]. Northwestern Polytechnical University, 2007

[3] Huai.Lin.Shu. PID neural network and its control system[M].National Defence Industry Press,2006.

[4] Yong.Wu, Shou.Sheng.Xie. Aero engine PID decoupling control based on support vector machine.[J]. Journal of Aerospace Power, 2009.24(10) :2349 2355.

[5] Kai.Long.Cai,Shou.Sheng.Xie,Ji.Ye.Wang.Aeroengine neural network decoupling control based on RBF Network Journal of Aerospace Power[J]. Propulsion technology, 2008,29(4):471 476

[6] Zhang Jing. Application of MATLAB in the control system[M]. Electronic Industry Press, 2007 